\title{
New echinocandin susceptibility patterns for nosocomial Candida albicans in Bogotá, Colombia, in ten tertiary care centres: an observational study
}

Giovanni Rodríguez-Leguizamón ${ }^{1,3,4}$, Alessandro Fiori ${ }^{3,4}$, Katrien Lagrou ${ }^{5}$, María Antonia Gaona ${ }^{2}$, Milciades Ibáñez ${ }^{1}$ Manuel Alfonso Patarroyo ${ }^{1,6}$, Patrick Van Dijck ${ }^{3,4}$ and Arley Gómez-López ${ }^{6 *}$

\begin{abstract}
Background: Candida albicans remains as the first cause of nosocomial fungal infections in hospitals worldwide and its susceptibility pattern should be better described in our tertiary care hospitals.

Methods: This study aimed at identifying the caspofungin susceptibility pattern regarding nosocomial Candida albicans infection in ten tertiary care hospitals using the methodology proposed by CLSI M27-A3 and CLSI M27-S4, and its association with risk factors and clinical outcome. The approach involved descriptive research concerning the diagnosis of nosocomial infection during a 7-month period in 10 hospitals in Bogotá, Colombia. Associations were established using exact non-parametric statistical tests having a high statistical power (>95\%), suitable for small samples. The exact Mann Whitney test or Kruskall-Wallis non-parametric ANOVA tests were used for distributions which were different to normal or ordinal variables when comparing three or more groups. Multivariate analysis involved using binomial, multinomial and ordinal exact logistical regression models (hierarchical) and discrimination power was evaluated using area under the ROC curve.

Results: 101 nosocomial infections were found in 82,967 discharges, for a Candida spp. infection rate of 12.2 per 10,000 discharges, $30.7 \%$ caused by C. albicans, $22.8 \%$ by C. tropicalis, 20.8\% by C. parapsilosis, $19.8 \%$ by other Candida, $3 \%$ by C. krusei and 3\% by C. glabrata. Statistically significant associations between mortality rate and the absence of parenteral nutrition were found in multivariate analysis ( $\mathrm{OR}=39.746: 1.794-880.59395 \% \mathrm{Cl}: \mathrm{p}=0.020)$. The model's predictive power was $83.9 \%$, having an $85.9 \%$ significant prediction area $(69.5 \%-10095 \% \mathrm{Cl} ; \mathrm{p}=0.001)$.

Conclusions: Significant differences were found regarding susceptibility results when comparing CLSI M27-A3 to CLSI M27-S4 when shifting clinical break-point values. However, one nosocomial strain was consistent in having reduced susceptibility when using both guidelines without having been directly exposed to echinocandins beforehand and no mutations were found in the FKS1 gene for hot spot 1 and/or hot spot 2 regions, thereby highlighting selective pressure regarding widespread antifungal use in tertiary healthcare centres. Nutritional conditions and low family income were seen to have a negative effect on survival rates.
\end{abstract}

Keywords: Candida albicans, Nosocomial infection, Susceptibility, Echinocandins, Resistance

\section{Background}

Candida spp. is the leading opportunistic mycotic agent worldwide and it is the fourth cause of bloodstream infections since the 1990s [1,2]. If the rate of mortality due to nosocomial candidaemia were to be specifically noted, it would have given a $49 \%$ attributable mortality

\footnotetext{
* Correspondence: arley.gomez.lo@gmail.com

${ }^{6}$ Molecular Biology and Immunology Department, Fundación Instituto de Inmunología de Colombia (FIDIC), Carrera 50\#26-20, Bogotá, Colombia Full list of author information is available at the end of the article
}

rate (38\%-60\% 95\% CI), this being very high for an infectious disease, even surpassing the patients' base disease mortality rate [3].

Continuous advances in medical techniques have also imposed fresh challenges on the management and control of nosocomial fungal infections [4]. According to reports collected over a six-and-a-half-year period of epidemiological surveillance at global level by the ARTEMIS study, C. albicans resistance to azoles was distributed as follows: 
1.3\% fluconazole resistance and $1.0 \%$ to voriconazole (however, the figures for Colombia are $6.1 \%$ for fluconazole and $4.0 \%$ for voriconazole) [5]. Regarding the echinocandins, global studies have been found which have not considered the level of resistance to be significant, given that echinocandins have only recently been introduced into clinical practice [6].

The variability in minimum inhibitory concentration (MIC) (break-points reported in different studies) [6-8], as well as clinical reports of therapeutic failure in patients having MIC results of susceptible strains $[9,10]$ have led to suggesting that break-points for defining susceptibility against echinocandins should be modified [11]. These antecedents motivated our group's interest in determining nosocomial C. albicans strains' susceptibility pattern in tertiary care hospitals in Bogotá, Colombia.

\section{Methods}

\section{Study design}

This observational study was carried out in ten tertiary care hospitals (each having between 200 and 500 hospital beds); three of these hospitals admit paediatric population only, whilst the remaining ones admit the general population. The nosocomial infection rate was $3.38 \%$ in non-ICU wards and $8.26 \%$ in ICUs; samples were collected during seven months, giving a total of 77,763 regular hospital discharges in non-ICU ward and 5,204 ICU discharges. The ICUs were mixed purpose (i.e. caring for surgical, coronary, septic and poly-traumatised patients). All cases of nosocomial Candida spp. infection were reported by infection committees and according to the CDC definition [12]. Ethics committees from the following institutions approved this study: Clínica del Niño, Clínica de Occidente, Fundación Cardioinfantil, Hospital de Kennedy, Fundación Homi-Hospital de la Misericordia, Hospital del Tunal, Hospital Simón Bolívar, Hospital de la Samaritana, Clínica Infantil Colsubsidio, Clínica Colsubsidio Orquídeas and Universidad del Rosario.

All the clinical records were reviewed by each hospital's nosocomial infection committee regarding patients reported as being cases of nosocomial Candida spp. infection during 7 months and for whom prior culture results were available. Evident infection 48 hours after having been admitted to hospital supported by a positive microbiological culture was defined as a case of nosocomial infection, registered in the clinical record by the treating physician. The treating physician defined whether the clinical diagnosis gave either invasive candidiasis or urinary tract infection by Candida spp. based on clinical interpretation, laboratory reports and positive cultures [12]. Clinical records were verified by medical researchers for all patients and the information was recorded in an Excel database and Epi Info (version 6.0). All clinical samples were collected as part of standard patient care and later managed by a centralised laboratory for verifying Candida spp. presence.

\section{Antifungal susceptibility test}

A broth micro-dilution test (BMD) was carried out at KU Leuven's Molecular Cell Biology laboratory according to the indications given in the CLSI M27-A3 document [13]. The new recommendations made in CLSI M27-S4 guidelines were incorporated in additional assays; such assays were carried out in the Corporación de Investigaciones Biológicas (CIB) microbiology laboratory in Medellín, following all guideline quality and reproducibility recommendations [14].

\section{Quality control}

Quality was controlled according to CLSI indications using recommended C. krusei ATCC 6258 and C. parapsilosis ATCC 22019 strains [13].

\section{Antifungal drugs}

Caspofungin (Merck), micafungin (Astellas) and fluconazole (Sigma) were used in this study. The working solution for each antifungal drug was prepared in sterile water following CLSI M27-A3 guidelines, the first two dilutions in water and the final dilutions in RPMI 1640 solution adjusted to $\mathrm{pH} 7.0$ with $0.165 \mathrm{M}$ morpholinepropanesulfonic acid (MOPS) buffer [13]. All C. albicans nosocomial isolates were tested with micafungin dissolved in DMSO, following CLSI M27-S4 recommendations.

\section{Microorganisms}

One hundred and one nosocomial Candida spp. strains were isolated throughout the study, 31 of them being nosocomial C. albicans. These isolates were obtained from the epidemiological surveillance being carried out by each hospital infection committee and corresponded to nosocomial infection criteria; each infection recorded corresponded to an episode per patient.

\section{Identifying the microorganisms}

Colombian clinical isolates were initially typed using manual microbiological systems (API32C and germ tube) and those classified as being $C$. albicans were further confirmed by using MALDI Biotyper RTC software 3.0 (Bruker); the identification spectrum was obtained in duplicate and compared to the MSP reference spectrum, simultaneously selected from the BDAL software database (Bruker) and the CBS-KNAW library. The classification results obtained by MALDI-TOF-MS (spectrum identification $c f$ MSP) led to assigning a score in line with the equipment's requirements and classified as follows: firm identification of genus and specie $(\geq 2.0)$, reliable identification of genus (1.7-2.0) and non-reproducible identification $(\leq 1.7)$ [15]. 


\section{Analysing FKS1 gene sequencing}

FKS1 gene sequences were amplified using previously described primers for hot spot (HS) 1 (HS1) and 2 (HS2) regions: F 5' GTTCCACCAGTTTATACATTCC 3' and 5' ATGTCACTCTTGAGAATTGATC 3' R for HS1 and F 5' GCTCATGAAGCTATCATGTGTT 3' and 5' CAA GACAAACACCTAAACATCC 3' R for HS2 [10,16]. All reactions involved using $\mathrm{KAPA} \mathrm{HiFi}$ containing $0.3 \mu \mathrm{M}$ of each primer in a final $25 \mu \mathrm{L}$ volume. Thermal conditions were set as follows: denaturing at $95^{\circ} \mathrm{C}$ for $5 \mathrm{~min}$, followed by 35 cycles of denaturing at $98^{\circ} \mathrm{C}$ for 30 seconds, primer annealing at $54^{\circ} \mathrm{C}$ for 20 seconds and an extension step at $72^{\circ} \mathrm{C}$ for $1 \mathrm{~min}$ followed by a final extension step at $72^{\circ} \mathrm{C}$ for $5 \mathrm{~min}$. Attempts were made to characterise mutations on at least two different occasions, forming part of two subsequent biological repetitions; the amplification products were purified by using a Promega Wizard purification systems kit and then sent for sequencing in both directions ( $5^{\prime}$ and $\left.3^{\prime}\right)$ with a BigDye Terminator kit (Macrogen, Seoul, South Korea).

\section{Statistical analysis}

The data was processed using SPSS (version 20.0) and STATA (version 11.0) software.

Absolute and relative frequency distribution was expressed as percentages for qualitative variables and measurements of central tendency, the average and mean dispersion, the range and standard deviation were used for quantitative variables. The association of susceptibility and mortality with different factors, Fisher's exact test and likelihood ratio were evaluated for qualitative variables and Shapiro Wilk's test was used for evaluating normality in normal variables, Student's $T$-test for average differences for independent groups having homogenous or heterogeneous variability, using Levene's test for evaluating the homogeneity of variance. The exact Mann Whitney test or Kruskall-Wallis non-parametric ANOVA tests were used for distributions which were different to normal or ordinal variables when comparing three or more groups. Multivariate analysis involved using binomial, multinomial and ordinal exact logistical regression models (hierarchical) and discrimination power was evaluated using area under the ROC curve. Statistical tests were evaluated using 5\% and $10 \%$ significance.

\section{Results}

\section{Patients' characteristics}

One hundred and one nosocomial Candida spp. infections were identified during 7 months from 77,763 nonICU ward discharges and 5,204 ICU discharges, giving an incidence rate of 12.2 nosocomial fungaemia per 10,000 discharges; distribution was $30.7 \%$ C. albicans, $22.8 \%$ C. tropicalis, $20.8 \%$ C. parapsilosis, $19.8 \%$ other Candida spp., 3\% C. krusei and 3\% C. glabrata. The distribution of nosocomial $C$. albicans infection showed that 7 clinical isolates (13\%) came from non-ICU ward discharges and 24 clinical isolates (87\%) from ICUs; Table 1 shows the related clinical characteristics. No significant differences were found regarding gender distribution (51.6\% females and $48.4 \%$ males; $\mathrm{p}=1.00$, exact binomial test). In age groups known as being at risk of acquiring infection, 8 patients (26\%) aged less than 1 year old and 7 patients (22\%) older than sixty were found. There was 33\% mortality, similar to that described in the literature [3]. Diagnosis for nosocomial infection caused by Candida spp. had a similar distribution between invasive candidiasis (58.1\%) and urinary tract infection (41.9\%) caused by C. albicans. Regarding risk factors, more than four broad-spectrum antibiotics were being used at the same time (10 patients: $32.3 \%$ ), central venous catheter use (25 patients: $80.6 \%)$, parenteral nutrition (18 patients: $58.1 \%$ ) and more than 15 days' hospital length of stay (27 patients: $87 \%$ ).

\section{Antifungal susceptibility}

Table 2 shows MIC values for determining caspofungin susceptibility following the standardised antifungal susceptibility test, according to CLSI M27-A3 indications for the $C$. albicans group previously identified by MALDITOF-MS. Table 2 also shows the MIC values used for determining micafungin susceptibility, according to the new CLSI M27-S4 recommendations. The GV608 strain was resistant to micafungin following the CLSI M27-S4 guidelines and had previously shown resistance to caspofungin following CLSI M27-A3 guidelines.

\section{Characterising the FKS1 gene mutation pattern}

The sequencing results were analysed using CLUSTALW software and compared to GenBank reference sequences

\section{Table 1 Demographic and clinical characteristics}

\begin{tabular}{lll}
\hline Variable & $\begin{array}{l}\text { ICU } \mathbf{n} \\
\text { cases (\%) }\end{array}$ & $\begin{array}{l}\text { Non-ICU ward } \\
\mathbf{n} \text { cases (\%) }\end{array}$ \\
\hline Age $\leq \mathbf{1}$ year & $7(23 \%)$ & $1(3 \%)$ \\
Age $\geq \mathbf{6 0}$ years & $5(16 \%)$ & $2(6 \%)$ \\
Male gender & $13(42 \%)$ & $2(6 \%)$ \\
Invasive Candidiasis & $14(45 \%)$ & $4(13 \%)$ \\
UTI & $10(32 \%)$ & $3(10 \%)$ \\
Mortality & $7(23 \%)$ & $3(10 \%)$ \\
Broad spectrum AB use & $24(77 \%)$ & $6(19 \%)$ \\
CVC & $21(68 \%)$ & $4(13 \%)$ \\
Abdominal surgery & $6(19 \%)$ & $4(13 \%)$ \\
TPN & $15(48 \%)$ & $3(10 \%)$ \\
Immunosuppressant & $6(19 \%)$ & 0 \\
Length of stay $\geq 15$ days & $22(71 \%)$ & $5(16 \%)$ \\
\hline
\end{tabular}

Note: ICU: intensive care unit, UTI: urinary tract infection, AB: antibiotic, CVC: central venous catheter, TPN: total parenteral nutrition. 


\begin{tabular}{|c|c|c|}
\hline Strain & MIC CSF & MIC MCF \\
\hline SC5314 & $\leq 0.25 \mu \mathrm{g} / \mathrm{mL}$ (S) & $\leq 0.25 \mu \mathrm{g} / \mathrm{mL}(\mathrm{S})$ \\
\hline GV475 & $\geq 1 \mu \mathrm{g} / \mathrm{mL}(\mathrm{R})$ & $\leq 0.25 \mu \mathrm{g} / \mathrm{mL}(\mathrm{S})$ \\
\hline GV78 & $0.5 \mu \mathrm{g} / \mathrm{mL}(\mathrm{l})$ & $\leq 0.25 \mu \mathrm{g} / \mathrm{mL}(\mathrm{S})$ \\
\hline GV527 & $2 \mu \mathrm{g} / \mathrm{mL}(\mathrm{R})$ & $\leq 0.25 \mu \mathrm{g} / \mathrm{mL}(\mathrm{S})$ \\
\hline GV242 & $\geq 1 \mu \mathrm{g} / \mathrm{mL}(\mathrm{R})$ & $\leq 0.25 \mu \mathrm{g} / \mathrm{mL}(\mathrm{S})$ \\
\hline GV147 & $\geq 1 \mu \mathrm{g} / \mathrm{mL}(\mathrm{R})$ & $\leq 0.25 \mu \mathrm{g} / \mathrm{mL}$ (S) \\
\hline GV608 & $\geq 1 \mu \mathrm{g} / \mathrm{mL}(\mathrm{R})$ & $0.5 \mu \mathrm{g} / \mathrm{mL}(\mathrm{R})$ \\
\hline
\end{tabular}

Note: MIC: minimal inhibitory concentration, CSF: caspofungin, MCF: micafungin, S: susceptible, I: intermediate, R: resistant.

The isolate resistant to both caspofungin and micafungin is shown in bold.

(accession No. D88815). No mutations were found in the GV608 nosocomial strain for the aforementioned regions after analysing both resistant strain sequences.

\section{Statistical association}

There was a significant association between mortality and low socioeconomic level, mortality being greater in patients having a daily income of less than $9 €(50.0 \%, 9 /$ 18 cf $15.4 \%, 2 / 13)(\mathrm{p}=0.05$, Fisher's exact test). C. albicans infected patients with no parenteral nutrition had a significant association with mortality when compared to those having parenteral nutrition $(61.8 \%, 8 / 13$ cf $16.7 \%$, $3 / 18)$ ( $\mathrm{p}=0.014$, Fisher's exact test).

The multivariate exact unconditional logistical regression model for mortality was significantly associated with the absence of parenteral nutrition $(\mathrm{OR}=39.746$ : 1.794- 880.593 $95 \% \mathrm{CI} ; \mathrm{p}=0.020$ ) and closely associated with a background of being undernourished $(\mathrm{p}=0.079, \mathrm{OR}=14.21)$. The other factors were not significant (having an abdominal surgery during hospital stay, the number of antibiotics or length of hospital stay). The model's discrimination power was $83.9 \%$ (85.9\% area under the ROC curve: $69.5-100$ 95\% CI; p = 0.001).

No statistically significant associations were found between the susceptibility pattern and the clinical outcomes.

\section{Discussion}

The present study explored the $C$. albicans infection pattern regarding nosocomial infection and led to characterising susceptibility patterns in a Colombian hospital setting. This was the first study of its kind to be carried out in a tertiary care hospital network in Bogotá; it also explored the echinocandin susceptibility profile as a recent therapeutic alternative for managing patients at risk of infection by this fungus. There were differences between CLSI M27-A3 and CLSI M27-S4 guidelines as the clinical break-points shifted. CLSI M27-S4 gave less strains having reduced susceptibility; however, a nosocomial strain was consistently micafungin-resistant, without having been directly exposed to echinocandins.

The other relevant findings concerned the clinical associations found regarding this type of nosocomial infection, as well as aspects related to the conditions of patients requiring nutritional support and having a low monthly income background, thereby affecting the clinical outcome.

Our study focused on providing a description of Candida spp. nosocomial infection; a high incidence rate of 12.2 cases of infection per 10,000 discharges was revealed in a representative sample of 10 tertiary care hospitals, accounting for 82,967 total discharges over a 7-month period. This finding contrasted with that in the pertinent literature which referred to nosocomial fungaemia incidence of 4.9 cases of infection per 10,000 discharges [17], thereby revealing greater nosocomial infection incidence caused by this microorganism in the sample of hospitals in Bogotá. Taking the total amount of nosocomial infections caused by Candida spp., distribution by species maintained the frequency described in previous studies in Colombia, where Candida albicans occupied first place regarding frequency (30.7\%) [18]; however, this is the first report made in Colombia which has evaluated clinical and microbiological aspects referring to a representative population involved in nosocomial infection from the 10 tertiary care hospitals which participated in the study.

The echinocandins are currently first-line treatment agents when managing patients having had previous exposure to azoles, a report of Candida spp. involving reduced susceptibility to azoles and/or patients in a critical or unstable clinical condition [19]. Caspofungin is the drug of choice for patients having the aforementioned characteristics; its use is widespread due to it being the first molecule approved by the FDA $[20,21]$.

According to epidemiological surveillance studies carried out around the world, excellent levels of activity for echinocandins in vitro have been observed during a six year follow-up, suggesting that emergent resistance to echinocandins is hardly likely since the caspofungin inhibition percentage for C. albicans was 99.6\% [22]. However, later studies have shown the need for re-evaluating the breakpoints defining echinocandin susceptibility as clinical observations have been made regarding therapeutic failure, in spite of susceptibility tests having indicated lower than $2 \mu \mathrm{g} / \mathrm{mL}$ values. Such break-points were adjusted for defining susceptibility as $\leq 0.25 \mu \mathrm{g} / \mathrm{mL}$, intermediate susceptibility as $0.5 \mu \mathrm{g} / \mathrm{mL}$ and $\geq 1 \mu \mathrm{g} / \mathrm{mL}$ for resistance [11]. A recent review of clinical break-points proposed that just $\leq 0.25 \mu \mathrm{g} / \mathrm{mL}$ should be used for susceptibility and $\geq 0.5 \mu \mathrm{g} / \mathrm{mL}$ for resistance [23]. Micafungin or anidulafungin use has recently been proposed instead of caspofungin in susceptibility tests because of variability regarding the break-points obtained by several laboratories [24]. Caspofungin is the most used antifungal 
agent in clinical practice and the correlation between in vitro break-points for micafungin regarding patients being treated with caspofungin thus merits new studies. Our study revealed differences between both CLSI guidelines, indicating that the M27-S4 guideline recommendations should be followed in new studies. It is worth stressing that just one nosocomial strain was consistently micafungin-resistant regarding both methodologies and had not been previously exposed to echinocandins. It should be noted that mutations in the FKS1 gene for HS1 and/or HS2, as described in the pertinent literature, were not found for this nosocomial strain [10]. The foregoing shows the need for adjusting clinical break-points, as the susceptibility pattern regarding Candida spp. infections is dynamic and is influenced by patient and strain exposure to prolonged treatment with different antifungal drugs currently available on the market $[9,10,25]$. Even though the molecular definition of resistance to echinocandins has led to identifying punctual mutations in the FKS1 gene $[16,20,26]$, other authors have managed to identify low susceptibility to echinocandins in the absence of such mutations $[27,28]$. The foregoing involves other mechanisms, meaning that other alternatives could be considered for explaining the reduced susceptibility pattern observed in our isolate $[27,28]$. This C. albicans strain could have represented a nosocomial infection transmission pattern where, according to prior reports, healthcare workers hands represent the main vehicle of transmission [29].

It is worth noting that this is the first observational study reported in a population having a higher nosocomial fungal infection rate $(12.2 / 10,000$ discharges) than that reported in pertinent literature $(4.9 / 10,000$ discharges [17]) which highlights the importance of the results here found, despite the relatively low sample size. However, associations were established using exact non-parametric statistical tests having a high statistical power (>95\%), suitable for small sample sizes $[30,31]$ to overcome this problem.

This is the first study which has evaluated a representative nosocomial sample of C. albicans infection and the first aimed at adapting and parametrising the CLSI M27-S4 guidelines for Colombia. Neither case had a report of having received prior antifungal treatment and this also had not been described previously in the literature; these results reinforce the concept of nosocomial fungal transmission and the microbiological outcomes highlight the need of carrying out combination drug therapy studies in order to minimise resistance risk [32,33]. Additional microbiological and molecular studies are required for describing this susceptibility pattern; this would lead to a better understanding of the mechanisms facilitating these microorganisms becoming adapted to exposure to such antifungal drugs.
Another relevant aspect concerning studying C. albicans infection concerns the clinical factors associated with a risk of contracting the infection [34]. In spite of some reports having indicated an increase in infections caused by non-albicans species, C. albicans continues being responsible for most cases involving fungal infections in hospitals [35], besides, the associated risk factors have not changed. The overall description of patients suffering C. albicans infection in our study has shown that the presence of central venous catheter, prior broad spectrum antibiotic use and parenteral nutrition had the greatest association [36], as well as most patients having stayed in an ICU.

Regarding the statistical analysis of 31 patients having nosocomial C. albicans infection, significant associations were identified which were related to the two most relevant variables: mortality and nutritional condition.

This study revealed an association between mortality and patients having a daily income of less than $9 €$, this being difficult to find in the pertinent literature and represents an external factor concerning the quality of hospital care which could affect an outcome which is unfortunately associated with a particular social condition. The Colombian healthcare system offers equal coverage to all population. Associated factors such as the type of institution or haemodynamic state which could have affected or been associated with this variable were also explored but their values were not significant (i.e. the level of medical treatment was equitable for all patients).

Regarding bivariate analysis of patients infected by $C$. albicans, another strongly associated factor referred to the absence of parenteral nutrition and mortality when contrasting our study with the literature related to mortality studies. This factor was not specifically mentioned [3], though references were made to the presence of parenteral nutrition and a risk of candidaemia [37]. However, some important work refers to nutritional state and infection and how compromise regarding nutritional state favours the risk of infection and even a patient becoming immunocompromised [38]. Multivariate analysis covering all the risk factors for mortality and candidaemia described in different work [3,37] was taken into account when designing an exact unconditional logistical regression multivariate model which led to finding a significant association between the absence of parenteral nutrition. This indicated the relevance of including patients' nutritional state and the relevance of including suitable nutritional support for providing their needs in a clinical evaluation.

Several studies have been published about nosocomial Candida spp. infection presentation mode and frequency, as well as regarding the risk factors associated with the infection [3,37]; however, little information is available regarding patients' susceptibility, socioeconomic conditions 
and nutritional support. The nosocomial infection rate found in the present study was representative of the samples taken in the tertiary care hospitals which participated in the study and was even higher than that reported in the literature [17]. The relevance of these results has emphasised aspects regarding prevention and the rational use of antifungal therapeutic schemes, expressed by other authors as being one of the best strategies for controlling this emergent problem [39,40].

\section{Conclusions}

Some differences were found regarding susceptibility results comparing CLSI M27-A3 to CLSI M27-S4 when shifting clinical break-point values. However, one nosocomial strain was consistent in having reduced susceptibility when using both guidelines without having been directly exposed to echinocandins beforehand and had no mutations in regions previously described for the FKS1 gene, thereby indicating selective pressure concerning widespread antifungal use in tertiary healthcare centres. Nutritional conditions and low family income were seen to have a negative effect on survival rates. The clinical findings documented regarding the first echinocandinresistant nosocomial strain in Colombia which has not been exposed to this antifungal drug indicate the urgent need for epidemiological surveillance studies regarding susceptibility patterns following the latest CLSI M27-S4 recommendations.

\begin{abstract}
Abbreviations
CLSI: Clinical Laboratory Standards Institute; ROC Curve: Receiver Operating Characteristic Curve; MIC: Minimum Inhibitory Concentration; ICU: Intensive Care Unit; CDC: Centers for Disease Control and Prevention; BMD: Broth Micro-Dilution test; MOPS: Morpholinepropanesulfonic acid buffer; MALDI: Matrix-Assisted Laser Desorption/lonization; MSP: Main Spectrum; MALDI-TOF-MS: Matrix-Assisted Laser Desorption/lonization Time Of Flight Mass Spectrometry; FDA: Federal Drug Administration; UTI: Urinary Tract Infection; AB: Antibiotic; CVC: Central Venous Catheter; TPN: Total Parenteral Nutrition.
\end{abstract}

\section{Competing interests}

KL has received research grants from Gilead, MSD and Pfizer, received travel support from MSD, Pfizer and Gilead and received lecture honoraria from Gilead, MSD, and Pfizer; the remaining authors state that they have no conflict of interest to declare.

\section{Authors' contributions \\ GRL conceived the study, participated in its design, analysed data and drafted the manuscript. AF conceived the study, participated in its design, participated on the acquisition of laboratory data and critically reviewed the manuscript. KL conceived the study, participated in its design and critically reviewed the manuscript. MAG participated on acquisition of laboratory data and critically reviewed the manuscript. Ml carried out the statistical analysis and critically reviewed the manuscript. MAP conceived the study, participated in its design, analysed data and critically reviewed the manuscript. PVD coordinated the study, participated in its design, analysed data and critically reviewed the manuscript. AGL conceived the study, participated in its design and analysed data. All authors read and approved the final manuscript.}

\section{Acknowledgments}

This work was financed by the ERASMUS MUNDUS ERACOL programme, by the KU Leuven Research Fund, the Fund for Scientific Research Flanders (FWO WO.026.11 N and G.0804.11) and by funding from the Universidad del
Rosario. We greatly acknowledge technical-scientific support from the Fundación Instituto de Inmunología de Colombia (FIDIC) and Ana Kolecka from CBS-KNAW Fungal Biodiversity Centre, Yeast and Basidiomycete Research, Utrecht, The Netherlands. We would like to thank Ilse Palmans and Deborah Seys for their technical support, as well as Beatriz Gómez and Catalina de Bedout from the Corporación de Investigaciones Biológicas (CIB) in Medellín for facilitating CLSI M27-S4 standardisation and application. We would like to thank Jason Garry for translating the manuscript.

\section{Author details}

'School of Medicine and Health Sciences, Universidad del Rosario, Bogotá, Colombia. ${ }^{2}$ Faculty of Natural and Mathematical Sciences, Universidad del Rosario, Bogotá, Colombia. ${ }^{3}$ VIB Department of Molecular Microbiology, KU Leuven, Leuven, Belgium. ${ }^{4} \mathrm{KU}$ Leuven Laboratory of Molecular Cell Biology, Leuven, Belgium. ${ }^{5}$ Department of Microbiology and Immunology, KU Leuven, Leuven, Belgium. ${ }^{6}$ Molecular Biology and Immunology Department, Fundación Instituto de Inmunología de Colombia (FIDIC), Carrera 50\#26-20, Bogotá, Colombia.

Received: 16 January 2015 Accepted: 18 February 2015

Published online: 28 February 2015

\section{References}

1. Nucci M, Queiroz-Telles F, Tobon AM, Restrepo A, Colombo AL. Epidemiology of opportunistic fungal infections in Latin America. Clin Infect Dis. 2010;51(5):561-70.

2. Perlroth J, Choi B, Spellberg B. Nosocomial fungal infections: epidemiology, diagnosis, and treatment. Med Mycol. 2007;45(4):321-46.

3. Gudlaugsson O, Gillespie S, Lee K, Vande Berg J, Hu J, Messer S, et al. Attributable mortality of nosocomial candidemia, revisited. Clin Infect Dis. 2003;37(9):1172-7.

4. Pfaller MA, Diekema DJ. Epidemiology of invasive candidiasis: a persistent public health problem. Clin Microbiol Rev. 2007;20(1):133-63.

5. Pfaller MA, Diekema DJ, Rinaldi MG, Barnes R, Hu B, Veselov AV, et al. Results from the ARTEMIS DISK Global Antifungal Surveillance Study: a 6.5-year analysis of susceptibilities of Candida and other yeast species to fluconazole and voriconazole by standardized disk diffusion testing. J Clin Microbiol. 2005;43(12):5848-59.

6. Pfaller MA, Diekema DJ, Ostrosky-Zeichner L, Rex JH, Alexander BD, Andes D, et al. Correlation of MIC with outcome for Candida species tested against caspofungin, anidulafungin, and micafungin: analysis and proposal for interpretive MIC breakpoints. J Clin Microbiol. 2008;46(8):2620-9.

7. Pfaller MA, Boyken L, Hollis RJ, Messer SA, Tendolkar S, Diekema DJ. Global surveillance of in vitro activity of micafungin against Candida: a comparison with caspofungin by CLSI-recommended methods. J Clin Microbiol. 2006;44(10):3533-8.

8. Messer SA, Moet GJ, Kirby JT, Jones RN. Activity of contemporary antifungal agents, including the novel echinocandin anidulafungin, tested against Candida spp., Cryptococcus spp., and Aspergillus spp.: report from the SENTRY Antimicrobial Surveillance Program (2006 to 2007). J Clin Microbiol. 2009;47(6):1942-6.

9. Baixench MT, Aoun N, Desnos-Ollivier M, Garcia-Hermoso D, Bretagne S, Ramires S, et al. Acquired resistance to echinocandins in Candida albicans: case report and review. J Antimicrob Chemother. 2007;59(6):1076-83.

10. Laverdiere M, Lalonde RG, Baril JG, Sheppard DC, Park S, Perlin DS. Progressive loss of echinocandin activity following prolonged use for treatment of Candida albicans oesophagitis. J Antimicrob Chemother. 2006;57(4):705-8.

11. Pfaller MA, Diekema DJ, Andes D, Arendrup MC, Brown SD, Lockhart SR, et al. Clinical breakpoints for the echinocandins and Candida revisited: integration of molecular, clinical, and microbiological data to arrive at species-specific interpretive criteria. Drug Resist Updat. 2011;14(3):164-76.

12. Horan TC, Andrus M, Dudeck MA. CDC/NHSN surveillance definition of health care-associated infection and criteria for specific types of infections in the acute care setting. Am J Infect Control. 2008;36(5):309-32.

13. Wayne PA, CLSI. Reference method for broth dilution antifungal susceptibility testing of yeast; CLSI document M27-A3. 2008.

14. CLSI. Reference method for broth dilution antifungal suscetibility testing of yeasts; fourth informational supplement. CLSI Document M27-S4. Wayne, PA: Clinical and Laboratory Standards Institute; 2012. 
15. Bader O, Weig M, Taverne-Ghadwal L, Lugert R, Gross U, Kuhns M. Improved clinical laboratory identification of human pathogenic yeasts by matrix-assisted laser desorption ionization time-of-flight mass spectrometry. Clin Microbiol Infect. 2011;17(9):1359-65.

16. Park S, Kelly R, Kahn JN, Robles J, Hsu MJ, Register E, et al. Specific substitutions in the echinocandin target Fks1p account for reduced susceptibility of rare laboratory and clinical Candida sp. isolates. Antimicrob Agents Chemother. 2005:49(8):3264-73.

17. Beck-Sague C, Jarvis WR. Secular trends in the epidemiology of nosocomial fungal infections in the United States, 1980-1990. National Nosocomial Infections Surveillance System. J Infect Dis. 1993;167(5):1247-51.

18. Cortes JA, Reyes P, Gomez C, Buitrago G, Leal AL. Fungal bloodstream infections in tertiary care hospitals in Colombia. Rev Iberoam Micol. 2011:28(2):74-8.

19. Pappas PG, Kauffman CA, Andes D, Benjamin Jr DK, Calandra TF, Edwards Jr $J E$, et al. Clinical practice guidelines for the management of candidiasis: 2009 update by the Infectious Diseases Society of America. Clin Infect Dis. 2009;48(5):503-35.

20. Perlin DS. Resistance to echinocandin-class antifungal drugs. Drug Resist Updat. 2007;10(3):121-30.

21. Kartsonis NA, Nielsen J, Douglas CM. Caspofungin: the first in a new class of antifungal agents. Drug Resist Updat. 2003;6(4):197-218.

22. Pfaller MA, Boyken L, Hollis RJ, Kroeger J, Messer SA, Tendolkar S, et al. In vitro susceptibility of invasive isolates of Candida spp. to anidulafungin, caspofungin, and micafungin: six years of global surveillance. J Clin Microbiol. 2008;46(1):150-6.

23. Arendrup MC, Cuenca-Estrella M, Lass-Florl C, Hope WW. Breakpoints for antifungal agents: an update from EUCAST focussing on echinocandins against Candida spp. and triazoles against Aspergillus spp. Drug Resist Updat. 2013;16(6):81-95.

24. Espinel-Ingroff A, Arendrup MC, Pfaller MA, Bonfietti LX, Bustamante B, Canton $\mathrm{E}$, et al. Interlaboratory variability of Caspofungin MICs for Candida spp. Using CLSI and EUCAST methods: should the clinical laboratory be testing this agent? Antimicrob Agents Chemother. 2013;57(12):5836-42.

25. Hernandez S, Lopez-Ribot JL, Najvar LK, McCarthy DI, Bocanegra R, Graybill JR. Caspofungin resistance in Candida albicans: correlating clinical outcome with laboratory susceptibility testing of three isogenic isolates serially obtained from a patient with progressive Candida esophagitis. Antimicrob Agents Chemother. 2004;48(4):1382-3.

26. Garcia-Effron G, Park S, Perlin DS. Correlating echinocandin MIC and kinetic inhibition of fks1 mutant glucan synthases for Candida albicans: implications for interpretive breakpoints. Antimicrob Agents Chemother. 2009;53(1):112-22.

27. Drakulovski P, Dunyach C, Bertout S, Reynes J, Mallie M. A Candida albicans strain with high MIC for caspofungin and no FKS1 mutations exhibits a high chitin content and mutations in two chitinase genes. Med Mycol. 2011:49 (5):467-74.

28. Plaine A, Walker L, Da Costa G, Mora-Montes HM, McKinnon A, Gow NA, et al. Functional analysis of Candida albicans GPI-anchored proteins: roles in cell wall integrity and caspofungin sensitivity. Fungal Genet Biol. 2008;45 (10):1404-14.

29. Sydnor ER, Perl TM. Hospital epidemiology and infection control in acute-care settings. Clin Microbiol Rev. 2011;24(1):141-73.

30. Siegel S, Castellan NJ. Non parametric statistics for the behavioral science. 2nd ed. New York: McGraw - Hill Publishing Co; 1988.

31. Gibbons JD. Nonparametric statistical inference. New York: McGraw Hill; 1971.

32. Rogers TR. Antifungal drug resistance: limited data, dramatic impact? Int J Antimicrob Agents. 2006;27 Suppl 1:7-11.

33. Maubon D, Garnaud C, Calandra T, Sanglard D, Cornet M. Resistance of Candida spp. to antifungal drugs in the ICU: where are we now? Intensive Care Med. 2014;40(9):1241-55.

34. Hermsen ED, Zapapas MK, Maiefski M, Rupp ME, Freifeld AG, Kalil AC. Validation and comparison of clinical prediction rules for invasive candidiasis in intensive care unit patients: a matched case-control study. Crit Care (London, England). 2011;15(4):R198.

35. Diekema D, Arbefeville S, Boyken L, Kroeger J, Pfaller M. The changing epidemiology of healthcare-associated candidemia over three decades. Diagn Microbiol Infect Dis. 2012;73(1):45-8.

36. Yang SP, Chen YY, Hsu HS, Wang FD, Chen LY, Fung CP. A risk factor analysis of healthcare-associated fungal infections in an intensive care unit: a retrospective cohort study. BMC Infect Dis. 2013;13:10.
37. Ostrosky-Zeichner L, Pappas PG, Shoham S, Reboli A, Barron MA, Sims C, et al. Improvement of a clinical prediction rule for clinical trials on prophylaxis for invasive candidiasis in the intensive care unit. Mycoses. 2009;54(1):46-51.

38. Chandra RK. Impact of nutritional status and nutrient supplements on immune responses and incidence of infection in older individuals. Ageing Res Rev. 2004;3(1):91-104.

39. Diekema DJ, Pfaller MA. Nosocomial candidemia: an ounce of prevention is better than a pound of cure. Infect Control Hosp Epidemiol. 2004;25(8):624-6.

40. Sobel JD, Rex JH. Invasive candidiasis: turning risk into a practical prevention policy? Clin Infect Dis. 2001;33(2):187-90.

\section{Submit your next manuscript to BioMed Central and take full advantage of:}

- Convenient online submission

- Thorough peer review

- No space constraints or color figure charges

- Immediate publication on acceptance

- Inclusion in PubMed, CAS, Scopus and Google Scholar

- Research which is freely available for redistribution 